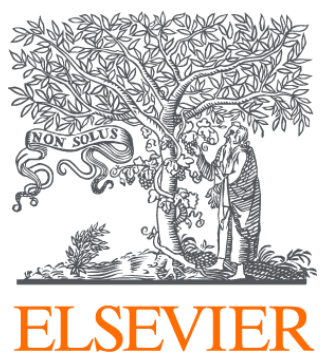

Since January 2020 Elsevier has created a COVID-19 resource centre with free information in English and Mandarin on the novel coronavirus COVID-

19. The COVID-19 resource centre is hosted on Elsevier Connect, the company's public news and information website.

Elsevier hereby grants permission to make all its COVID-19-related research that is available on the COVID-19 resource centre - including this research content - immediately available in PubMed Central and other publicly funded repositories, such as the WHO COVID database with rights for unrestricted research re-use and analyses in any form or by any means with acknowledgement of the original source. These permissions are granted for free by Elsevier for as long as the COVID-19 resource centre remains active. 
locations, or using a courier for collecting samples. The Experience category reflects the desire to stay involved if their experience is relaxed, hassle-free, and fun, with emphasis on the study being well-organized. Participants stressed the importance of Communication such as reminders, annual updates, and newsletters. Compensation was important to some participants such as gift cards, parking reimbursement, or non-monetary items such as thank you cards and tokens of appreciation. CONCLUSIONS: This study was co-created by PRPs who were actively involved throughout all stages of the project. The results from this patient-led study will help inform adaptations to recruitment and retention strategies for the national IMAGINE SPOR study.

\section{PATIENTS WITH INFLAMMATORY BOWEL DISEASE PERSPECTIVE ON COVID-19 AND HEALTH CARE SERVICE DURING THE PANDEMIC}

Randi Opheim, Kristian Moum, Bjørn Moum

BACKGROUND: Patients with inflammatory bowel diseases (IBD) have experienced changes to the routine management of their conditions during the coronavirus disease (COVID19) pandemic. The disease as well IBD treatment frequently require immunosuppressant medications, which could increase their risk of infection. The aim of this study was to determine patients' experience of the health care service, including the restrictions of hospitals visits made in Norway from Mars 12th 2020. METHOD: From June 18 to September 18 2020, all patients at the IBD outpatient clinic at Oslo University Hospital in Norway on biological therapy or other immunosuppressant's were included. A questionnaire including patients concerns regarding their disease, medical therapy and COVID-19, as well as their health care service needs in follow-up during the COVID-19 pandemic. RESULTS: Altogether 506 IBD patients answered a paperbased questionnaire. The mean age was 40.78 (SD 14.71), 289/506 (57\%) men, ulcerative colitis 199/506 (39\%), Crohn's disease 307/506 (61\%). Sixty-three patients $(12.5 \%)$ used biological therapy in combination with azathioprine or steroids. Ninety-one (18.2\%) were in obligated quarantine with negative test. Five patients $(4.9 \%)$ tested positive to SARS- CoV-2 of the 98 patients tested, (1.0\% of the total sample). One third of the IBD patients perceived they had increased risk for being infected by SARS- CoV-2 because of the immunosuppressive drugs they used. Nonetheless, 496/506 (98.6\%) of the patients adhered to continuing their medication. One-hundred and sixty-one $(32.3 \%)$ voluntarily isolated, and $21 / 506$ (4.2\%) was in sick leave being afraid of being infected. Furthermore, 20/506 $(4.0 \%)$ cancelled their consultation because they were afraid of being infected from SARS- CoV-2 at the hospital. The hospital changed physical consultation to telephone consultation for $75 / 506$ (15.0\%) of the patients. Thirtyeight patients $(7.6 \%)$ reported that they were afraid of going to the hospital because of restrictions due to the COVID-19 pandemic, and 18/506 (3.6\%) did not feel safe when at hospital. Approximately half of the IBD patients (219/506) were satisfied with the information provided by physician about medical treatment for IBD and Covid-19 while 398/ $506(77.3 \%)$ were satisfied with the information from health-care providers about restrictions due to COVID-19.
There were no statistical differences between Crohn's disease and ulcerative colitis. CONCLUSION: IBD patients on biological treatment and immunosuppressives took precautions because of fear of being infected with SARS- CoV-2. At the same time, they adhere to medical treatment regimens and follow-up at the hospital. Most patients were satisfied with the information they received from physicians and other health-care workers. One percent tested positive to SARS-CoV-2.

\section{RECENT APPROACHES FOR SMOKING CESSATION IN CROHN'S DISEASE: A QUALITATIVE REVIEW}

Jooyoung Moon, Hanna Moon

BACKGROUND: The clinical practice guideline for Crohn's disease (CD) management strongly recommends the avoidance and cessation of smoking. However, no standardized smoking cessation (SC) program exists for CD patients. Results from a nationwide survey among U.S. gastroenterologists have shown that SC counseling occurs irregularly and that almost half are not comfortable discussing SC strategies with their patients. These findings suggest that systematization and improvements in approaches for SC are necessary. This study aimed to provide a comprehensive review on recent developments and recommendations in SC care for CD patients. METHODS: A systematic literature review was conducted using PubMed, Google Scholar, and Web of Science databases for articles published from years 2016 to 2020. Two researchers independently screened and reviewed data. Inclusion criteria included peer-reviewed, published scientific articles written in the English language that examined SC in CD patients. Only studies with adults ( $\geq 18$ years old) and those with more than 10 subjects were included. No geographic limitations were applied. A total of 12 articles met our inclusion criteria. RESULTS: CD patients were found to have a significantly lower rate of SC when compared to the average population. Initial diagnosis of CD served as a strong motivator to quit, suggesting that SC education and intervention should begin on the first visit. Other significant positive predictors for SC in previous smokers included male gender, older age, and higher BMI. Adoption of a standardized program with a systematized process of questioning, documenting, and educating patients on SC resulted in an increase in discussion of SC strategies. Studies which expressed concerns regarding the deleterious effects of passive smoking on CD suggested that SC counseling be more broadly applied to include a patient's cohabitants. For those who require more intervention, addiction medicine consultation has been shown to reduce smoking use by a substantial margin of $27 \%$. In order to increase the success of SC, a recent trial has taken a personalized approach that involves measuring an individual's nicotine metabolite ratio to recommend a matching medication. Results have been promising with high rates of satisfaction of 85\%. CONCLUSIONS: Gastroenterologists should provide SC counseling more often and on a more regular basis, especially for patients who receive an initial diagnosis of CD. As existing SC strategies have limitations, new approaches are being actively explored, but further studies are desperately needed. Development of standardized and personalized SC programs should be considered so that SC counseling 